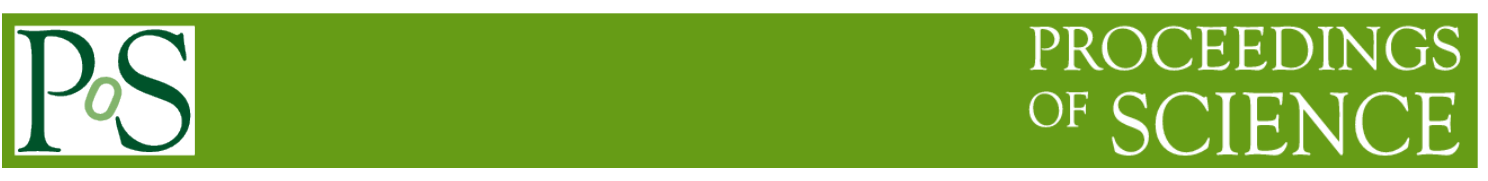

\title{
Challenges and Status of the ESSnuSB Accumulator Design
}

\author{
Ye Zou ${ }^{1}$ \\ Uppsala University \\ P.O Box 516, S-75120, Uppsala, Sweden \\ E-mail: ye.zouephysics.uu.se
}

The $2.0 \mathrm{GeV}, 5 \mathrm{MW}$ proton linac for the European Spallation Source, ESS, will have the capacity to accelerate additional pulses, interleaved with the proton pulses for neutron production, and send them to a neutrino target, providing an excellent opportunity to produce an unprecedented highperformance neutrino beam, the ESS neutrino Super Beam (ESSnuSB), to measure, with precision, the $\mathrm{CP}$ violating phase at the $2^{\text {nd }}$ oscillation maximum. In order to comply with the acceptance of the target and horn systems that will form the neutrino super beam, the long pulses from the linac must be compressed by about three orders of magnitude with minimal particle loss, something that will be achieved in an accumulator ring. This ring will accommodate about $10^{15}$ protons, which means that several design challenges are encountered. Strong space charge forces, low-loss injection, efficient collimation, and e-p instabilities are some of the aspects central to the design work. Different pulse structures and injection painting schemes have been studied, with the goal of mitigating space charge effects and of minimizing the heating of the stripping foil despite the very high beam intensity. This paper presents the status of the accumulator ring design, with multi-particle simulations of the injection procedure.

The 20th International Workshop on Neutrinos (NuFact2018)

12-18 August 2018

Blacksburg, Virginia

\section{$1 \quad$ Speaker}




\section{The ESSnuSB introduction}

The European Spallation Source (ESS) [1] currently being built in Lund, Sweden, will be one of the largest science and technology facilities in Europe. It will provide the world's most powerful neutron source, based on a $5 \mathrm{MW}$ proton beam. The superconducting ESS linac will accelerate $2.86 \mathrm{~ms}$ long pulses of protons to $2 \mathrm{GeV}$ at $14 \mathrm{~Hz}$, each pulse containing $1.1 \times 10^{15}$ protons, will be led to the neutron target station, impinging on the target to produce huge amount of spallation neutron source. That means that the duty cycle of the ESS linac is $4 \%$, while the RF cavities in the linac can accept up to $10 \%$ duty cycle. So, by doubling the pulse repetition frequency, the ESS linac can, with moderate modifications, be used to accelerate and deliver additional pulses for a neutrino facility [2]. For the current design of the neutrino target station, which is based on the EURO $v$ study, the magnetic horn requires a maximum pulse duration of 1.5 $\mu s$ due to ohmic heating in the horn. This means that to the $2.86 \mathrm{~ms}$ pulse from the linac must be compressed by three orders of magnitude in an accumulator ring with about $400 \mathrm{~m}$ circumference, a ring that will be added to the ESS complex.

\section{Challenges in the accumulator design}

In the design of an accumulator which can accommodate up to $5 \mathrm{MW}$ average beam power, the primary concern is the radioactivation caused by excessive uncontrolled beam loss, which can limit a machine's availability and maintainability. Based on the experience from the Spallation Neutron Source and other high-power high-intensity facilities, the hands-on maintenance demands an average uncontrolled beam loss not exceeding $1 \mathrm{~W} / \mathrm{m}$. At the $5 \mathrm{MW}$ level, that implies a fractional beam loss of $2 \times 10^{-7}$ per meter, or a total of $10^{-4}$ fractional uncontrolled beam loss for the whole accumulator.

Uncontrolled beam loss usually comes from a high space charge tune shift, beam injection, limited transverse and momentum acceptance, instabilities, and so on. One major contribution for beam loss is the tune shift due to direct space charge in the high intensity beam. A first estimate of the effect can be given by the direct tune shift formula,

$$
\Delta Q_{x, y}=\frac{-r_{0} N}{2 \pi \epsilon_{x, y} \beta^{2} \gamma^{3}} \frac{F}{B_{f}}
$$

where $r_{0}$ is the classical proton radius, $N$ the beam intensity, $\epsilon$ the emittance, $\beta^{2} \gamma^{3}$ the relativistic factors, $B_{f}$ the longitudinal bunching factor and $F$ the form factor depending on the particle distribution in the transverse plane, 1 for uniform, 2 for Gaussian.

The ESSnuSB parameters in Eq. (1)gives the value $\Delta Q_{x, y}=-0.5$ which is much higher than the generally accepted tune shift of -0.2 . Therefore, the linac beam pulse will be accumulated in 3 or 4 batches, thus limiting the total intensity in the ring by a factor $1 / 3$ or $1 / 4$. Direct proton injection, which is Liouvillean system, has been investigated for the ESSnuSB accumulator through simulations, assuming a tilted septum. The injection process is optimized for minimal beam loss on the septum. This optimization was done successfully for the ESSnuSB accumulator when space charge was ignored. When taking space charge into account, however, intolerable levels of beam loss on the septum are unavoidable. Currently, charge-exchange injection based on foil stripping, widely used for high-power, high-intensity proton accumulators, is studied for the ESSnuSB accumulator ring. However, foil scattering, heating, radiation, limited lifetime, handling incompletely stripped particles as well as the stripped electrons, are all demanding issues of the technique. As a first, the injection process is optimized to produce as few foil hits as possible 
while producing a best beam distribution from the point of view of reducing space charge effects in the ring. In addition, we are following the development of laser stripping at the SNS and JPARC [3, 4]. Collimation system is adopted to localize the controllable beam loss. For an accumulator with $5 \mathrm{MW}$ beam power, higher than $90 \%$ collimation efficiency is required, which needs sufficient transverse and momentum acceptance. Besides, the e-p instabilities could also cause uncontrolled beam losses, which should be well considered.

\section{Status of the accumulator design}

Different pulse structure schemes in the ESS linac have been considered [5] mainly to mitigate the space charge tune shift during the injection process to the ring. The case of $2.86 \mathrm{~ms}$, $50 \mathrm{~mA} \mathrm{H}^{-}$pulse with $14 \mathrm{~Hz}$, injected into one ring, with three gaps of $100 \mu$ s for the preparation of each filling with laser-stripping injection, and with 100 ns gaps for extraction, is the baseline operation scheme. The lattice, $384 \mathrm{~m}$ in circumference with 4-fold symmetry, FODO structure in the arcs, and four long straight sections for beam injection, collimation, extraction, and RF system, respectively, has shown good performance with very small beta beating. This lattice is designed for foil-stripping, which means it must be modified to accommodate the laser-stripping in the baseline scenario. However, the lattice, assuming foil stripping, is used in the first multi-particle simulations with the code PyORBIT with PTC external libraries. The injected beam distribution is Gaussian with $0.25 \pi \mathrm{mm}$ mrad normalized rms emittance in the transverse plane, uniform distribution with $15 \%$ gap in the longitudinal plane and the energy distribution is Gaussian with $0.1 \% \mathrm{rms}$ spread. 1000 macro particles per turn are injected, and injection takes place during 529 turns. A 2D FFT transverse space charge node is added with a grid of $128 \times 128$ bins, weighted with the longitudinal beam density, while FFT longitudinal space charge node also added with 128 bins. Figure 1 shows the first results of the transverse beam profile from correlated painting with and without space charge, from which one can see clearly increase of the emittance and the beam tail driven by space charge, which also causes inner beam with very small action. More simulations, including anti-correlated painting, will be done in the future.
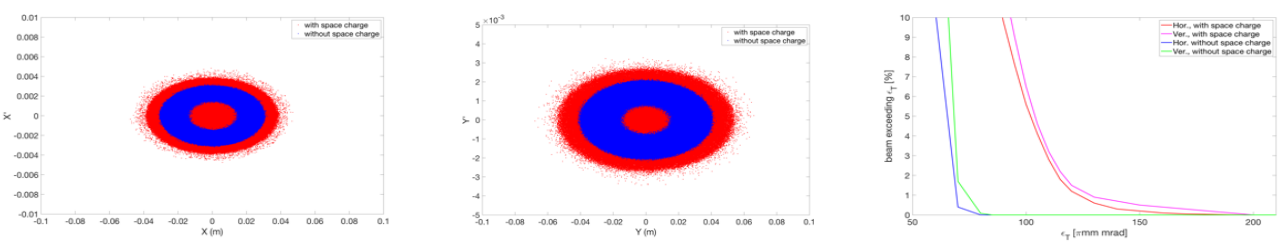

FIG. 1. Transverse beam profile from correlated painting (tunes: $8.27,8.37$ ) and the beam tail.

\section{References}

[1] S. Peggs (Ed.), ESS Technical Design Report, ESS-doc-274, 2013.

[2] E. Wildner et.al., Advances in High Energy Physics, Hindawi Publishing Corporation, Volume 2016, Article ID 8640493, http://dx.doi.org/10.1155/2016/8640493

[3] Sarah Cousineau et.al., PRL 118, 074801 (2017)

[4] P. K. Saha et.al., Proceedings of the 14th Annual Meeting of Particle Accelerator Society of Japan, PASJ2017 WEP024, p. 866, August 1-3, 2017

[5] M. Olvegård et.al., Overview of the ESSnuSB Accumulator Ring, Proceedings of HB2016, Malmö, Sweden, p.105, 2016. 